\title{
ENERGY-LIKE LIAPUNOV FUNCTIONALS FOR LINEAR ELASTIC SYSTEMS ON A HILBERT SPACE*
}

BY

\author{
J. A. WALKER
}

(Northwestern University)

\begin{abstract}
An approach is presented for generating energy-like functionals for linear elastic dynamic systems on a Hilbert space. The objective is to obtain a family of functionals which may be used for stability analysis of the equilibrium; i.e., Liapunov functionals. Although the energy functional, when one exists, is always a member of this family, the family is shown to exist even when an energy functional does not. Several discrete and distributed-parameter examples are presented, as are certain specific techniques for utilizing this approach.

1. General problem. The physical problems which led to the present work are in the area of stability of equilibria of linear elastic nonconservative systems. Such problems may be placed in the following general framework. Consider a real Hilbert space $\mathfrak{H C}$, independent of time, possessing a suitable inner product $\langle$,$\rangle , and a dynamical system$ which is assumed well-defined by the evolution equation

$$
\hat{M} \ddot{y}(t)+\hat{C} \dot{y}(t)+\hat{K} y(t)=0,
$$

under the condition that at every fixed $\tau \in[0, \infty)$ the state $(y(\tau), \dot{y}(\tau)) \in \mathcal{Y} \times \mathcal{Y}$, where

(i) $(\cdot)$ denotes $d / d t$ and the time $t \in[0, \infty)$,

(ii) $\mathcal{Y}$ is a linear manifold dense in $\mathcal{H C}$ and independent of time,

(iii) the real linear operators $\hat{M}, \hat{C}, \hat{K}$ are independent of time, $\mathscr{D}_{\hat{M}}=\Re_{\hat{M}}=\Re_{\hat{K}}=\mathfrak{F}$, $D_{C}=D_{\hat{K}}=\mathcal{Y}, R_{C} \subset \mathfrak{H}$,

(iv) $\hat{M}^{-1}$ and $\hat{K}^{-1}$ exist.
\end{abstract}

Here the symbols $D_{A}$ and $\Omega_{A}$ denote the domain and range, respectively, of an operator $A$.

This description of the system to be discussed is purposely not too specific. The approach to be presented here is applicable to a large class of elastic systems which cuts across the usual mathematical classifications, such as ordinary differential equations or partial differential equations, and is instead limited by linearity, being autonomous, being second-order in time, and allowing arbitrary initial conditions $(y(0), \dot{y}(0))$ in the state space $\mathcal{Y} \times \mathcal{Y}$ with an isolated equilibrium at $(y, \dot{y})=(0,0)$. The objects represented by the symbols $y, \hat{M}, \hat{C}, \hat{K}, \mathcal{Y}, \mathcal{H}$ will vary from one physical problem to another. The approach to be presented is intended for stability analysis of a broad class of elastic systems which share the above properties (linearity, etc.) but vary considerably in their mathematical description. Normally $y$ represents displacement (vector, function,

* Received July 11, 1971; revised version received January 20, 1972. This work was supported in part by the National Aeronautics and Space Administration under Grant No. NGR 14-007-067. 
etc.) and $\dot{y}$ represents velocity. The real Hilbert space $\mathcal{H C}$ is to be defined only after the system (1.1) has been mathematically classified, so as to specify an inner product and natural norm as well as the general class of elements for which this inner product has meaning. A desirable norm is one which has physical meaning, keeping in mind that the meaning of stability is often dependent upon the norm being used.

For an $n$-degree-of-freedom mechanical system, $y$ is a real $n$-vector of displacement, the operators $\hat{M}, \hat{C}, \hat{K}$ are $n \times n$ matrices, and $\mathcal{Y}=\mathcal{H} C=\mathcal{E}_{n}$ where $\mathcal{E}_{n}$ is the real $n$-dimensional Euclidean space having the inner product $\left\langle u_{1}, u_{2}\right\rangle \triangleq u_{1}^{T} u_{2}, u_{1} \in \mathcal{E}_{n}, u_{2} \in \mathcal{E}_{n}$. A problem of vibration of a mass continuously distributed over a bounded region $\Omega$ in $\mathcal{E}_{m}(m=1,2$ or 3$)$ is often described by a partial differential equation. In this case $y$ is usually the displacement of a point in $\Omega, \mathfrak{K C}$ is the space of real functions which are Lebesgue square-integrable over $\Omega, y$ is the subset of such functions which also satisfy given linear, time-independent, homogeneous boundary conditions on the boundary of $\Omega$ as well as "appropriate smoothness conditions", and $\hat{M}$ describes the spatial distribution of mass while $\hat{C}$ and $\hat{K}$ are linear spatial differential operators. None of this, however, is meant to imply that these are the only meanings which may be assigned to $y, \hat{M}$, $\hat{C}, \hat{K}, y, \mathfrak{z e}$.

The question of interest is the stability of the equilibrium $(y, \dot{y})=(0,0)$ for a general system of the form (1.1) satisfying (i) through (iv). Since the actual solutions of (1.1) are theoretically obtainable for arbitrary initial conditions by the methods of linear analysis, the stability question is readily answered-in theory. However, there is good reason for approaching the stability question from a Liapunov standpoint rather than that of direct solution.

Using eigenvalue analysis one is faced with the problem of examining $2 n$ eigenvalues even for a discrete $n$-degree-of-freedom system. Since this must normally be done numerically, the effects on stability of individual parameter changes become quite difficult to determine. In practice the operator $\hat{C}$ is usually ignored, effectively reducing the number of eigenvalues to $n$. In this case one finds that either all eigenvalues have zero real part or at least one has positive real part, implying that the equilibrium of (1.1) is either critically stable or unstable but never asymptotically stable. As is well known in stability theory [1], a prediction of critical stability on the part of the system model (1.1) implies nothing about the modeled system; it implies only that the model is a failure for the purpose of stability analysis.

This tendency to neglect the $\hat{C}$ operator when doing stability analysis via eigenvalue analysis has led to some seemingly odd results for "circulatory" systems $[2,3,4]$. On the other hand, the eigenvalue problem is several times more difficult if $\widehat{C}$ is not neglected. It would seem considerably more useful to utilize a method of stability analysis which is capable of detecting and predicting the effect of parameter changes, both small and large. The direct method of Liapunov is just such a method.

In the majority of physical problems in which (1.1) describes the motions of a mechanical system, $\hat{M}$ is found to be a symmetric operator: $\left\langle u_{1}, \hat{M} u_{2}\right\rangle=\left\langle\hat{M} u_{1}, u_{2}\right\rangle$ for all $u_{1}, u_{2} \in \mathcal{K}$. If $\hat{K}$ is also symmetric, (1.1) is termed a noncirculatory system, which implies the existence of an energy functional $E$ defined as

$$
2 E=\langle\dot{y}, \hat{M} \dot{y}\rangle+\langle y, \hat{K} y\rangle, \quad(y, \dot{y}) \in \mathcal{Y} \times \mathcal{Y}
$$

having the time derivative according to (1.1)

$$
2 \dot{E}=-2\langle\dot{y}, \hat{C} \dot{y}\rangle \text {. }
$$


If the energy functional exists and if $\hat{C}$ is a semidefinite operator, $2 E$ often serves admirably as a Liapunov functional and so may easily be used to determine stability. Further, the effect on stability of qualitative and quantitative changes in the $\hat{M}, \hat{C}, \hat{K}$ operators can be easily and directly determined from this particular form of Liapunov functional. In addition, $2 E$ becomes an integral if $\hat{C}=0$.

Unfortunately, for mechanical systems of recent interest (see Ref. [2]) the operator $\hat{K}$ is not symmetric and an energy functional does not exist, which is to say that the functional (1.2) does not have the derivative (1.3) and is not an integral for $\hat{C}=0$. Such systems are termed circulatory. Even for noncirculatory systems, the energy functional fails when $\hat{C}$ is indefinite. Our objective here is to devise a means of generating Liapunov functionals for both circulatory and noncirculatory systems and, in particular, Liapunov functionals which share some of the very desirable structural properties of the energy functional.

2. Construction of a Liapunov functional. Since $\hat{M}$ was assumed invertible, let us simplify our notation by defining $C=\hat{M}^{-1} \hat{C}, K=\hat{M}^{1} \hat{K}$, and consider (1.1) in the form

$$
\ddot{y}(t)+C \dot{y}(t)+K y(t)=0
$$

where at any fixed $\tau \in[0, \infty)$ the state $(y(\tau), \dot{y}(\tau)) \in \mathcal{Y} \times \mathcal{Y}$. Note that this new problem satisfies the same conditions, (i) through (iv), as (1.1) when $\hat{M}, \hat{C}, \hat{K}$ are replaced by $I, C, K$, and $I$ is the identity operator.

In order to generate candidates for Liapunov functionals with the simple structure of (1.2), even when $K$ is not symmetric, let us consider the functional

$$
V=\langle\dot{y}, G \dot{y}\rangle+\langle y, G K y\rangle, \quad(y, \dot{y}) \in \mathcal{Y} \times \mathcal{Y},
$$

which has as its time derivative according to (2.1) the functional

$$
\dot{V}=-2\langle\dot{y}, G C \dot{y}\rangle, \quad \dot{y} \in \mathcal{Y}
$$

provided the real linear operator $G$ satisfies the conditions

i) $G$ is symmetric, $\mathfrak{D}_{G}=\mathfrak{H}$,

ii) $G K$ is symmetric on $D_{G K}=y$.

We note that $V$ and $\dot{V}$ are similar to $2 E$ and $2 \dot{E}$ (when $K$ is symmetric) in their dependence on $y, \dot{y}$, and the operators $C$ and $K$. In fact, for noncirculatory problems we may choose $G=\hat{M}$ and produce $V=2 E$. However, the above formulation is also valid for circulatory systems provided a $G$ satisfying (2.4) exists. If for such a $G, \dot{V}$ is semidefinite in $\dot{y}, V$ is generally useful as a Liapunov functional to determine stability of the equilibrium $(y, \dot{y})=(0,0)$ of $(2.1)[5]$.

It is also of interest to note that the functional

$$
U=\langle\dot{y}+C y, G \dot{y}+G C y\rangle+\langle y, G K y\rangle, \quad(y, \dot{y}) \in \mathcal{Y} \times \mathcal{Y}
$$

has the time derivative for (2.1)

$$
\dot{U}=-2\langle G K y, C y\rangle, \quad y \in \mathcal{Y}
$$

provided $G$ satisfies (2.4). Thus $U$ is generally useful as a Liapunov functional provided a $G$ can be found such that $\dot{U}$ is semidefinite in $y$. We note that $\dot{U}$ is a functional of $y$ whereas $\dot{V}$ is a functional of $\dot{y}$, and $U=V$ iff $C=0$. 
Therefore, Liapunov functionals of the form $V$, or $U$, or some linear combination of $V$ and $U$, appear to be feasible for (2.1) provided operators $G$ exist satisfying (2.4). In particular, when (1.1) is noncirculatory we may choose $G=\hat{M}$ and so produce $V=2 E(U \neq 2 E$ unless $C=0)$, although we generally need not do so. That is, a set of $V$ (or $U$ ) functionals seems to be derivable; and the energy functional, when it exists, will be one and only one of them. For circulatory systems, of course, an energy functional does not exist. However, we still expect to produce a set of $V$ (or $U$ ) functionals. Before considering the general questions of existence and properties of $G$, we will illustrate what this approach is designed to do by applying it to lumped-parameter systems.

3. Lumped-parameter systems. In the case of lumped-parameter systems, $y$ is an $n$-vector, the operators $C$ and $K$ are $n \times n$ matrices, and $\mathcal{Y}=\mathscr{C}=\mathcal{E}_{n} . G$ is also an $n \times n$ matrix and conditions (2.4) require that both $G$ and $G K$ be symmetric. This results in no more than $n^{2}-n$ linear equations in the $n^{2}$ elements of $G$. Thus there normally are at least $n$ linearly independent matrices $G$ of the required form. A portion of the following is more fully discussed in [6], along with several examples, but several new results are presented here.

Utilizing the functional $V$ of (2.2),

$$
V=\dot{y}^{T} G \dot{y}+y^{T} G K y, \quad \dot{V}=-2 \dot{y}^{T} G C \dot{y},
$$

the following theorems can be proved [6]:

Theorem 1: If there exists a matrix $G$ such that $G C$ is positive definite, while $G$ and $G K$ are symmetric and positive definite, the equilibrium is asymptotically stable.

Theorem 2: If there exists a matrix $G$ such that $G C$ is non-negative, while $G$ and $G K$ are symmetric and positive definite, the equilibrium is stable.

Theorem 3: If there exists a matrix $G$ such that $G C$ is positive definite, while $G$ and $G K$ are symmetric but not both non-negative, the equilibrium is unstable.

Theorem 4: If there exists a matrix G such that GC is skew-symmetric, while $G$ and $G K$ are symmetric and definite of the same sign, the equilibrium is stable but not asymptotically stable.

Theorem 5: If there exists a matrix $G$ such that $G C$ is skew-symmetric, while $G$ and $G K$ are symmetric, the functional $V=\langle\dot{y}, G \dot{y}\rangle+\langle y, G K y\rangle$ is an integral of the motion.

Using the functional $U$, as well as $V$, the above theorems may be extended by replacing " $G C$ " with " $G C$ or $G K C$ " in the statement of each theorem.

To illustrate the application of this method to lumped-parameter systems, consider a system of the form (1.1) where

$$
\hat{M}=\left[\begin{array}{cc}
m_{1} & 0 \\
0 & m_{2}
\end{array}\right], \quad \hat{C}=\left[\begin{array}{ll}
c_{1} & c_{2} \\
c_{3} & c_{4}
\end{array}\right], \quad \hat{K}=\left[\begin{array}{cc}
k_{1} & -k_{3} \\
k_{3} & k_{2}
\end{array}\right]
$$

and $y$ is a real 2-vector. This represents a circulatory two-degree-of-freedom system, and an encrgy functional does not exist. In the form (2.1) we have

$$
\ddot{y}(t)+C \dot{y}(t)+K y(t)=0, \quad(y, \dot{y}) \in \varepsilon_{2} \times \varepsilon_{2},
$$

where $y=3 C=\varepsilon_{2}$ and

$$
C=\frac{1}{m_{1} m_{2}}\left[\begin{array}{ll}
m_{2} c_{1} & m_{2} c_{2} \\
m_{1} c_{3} & m_{1} c_{4}
\end{array}\right], \quad K=\frac{1}{m_{1} m_{2}}\left[\begin{array}{cc}
m_{2} k_{1} & -m_{2} k_{3} \\
m_{1} k_{3} & m_{1} k_{2}
\end{array}\right] .
$$


As pointed out in [6], it often appears to be advantageous to choose $\operatorname{diag}[G]=$ $\operatorname{diag}[\hat{M}]$ when $\hat{M}$ is a diagonal matrix. By (2.4) $G$ and $G K$ are to be symmetric, which implies

$$
G=\left[\begin{array}{cc}
m_{1} & g \\
g & m_{2}
\end{array}\right], \quad g=\frac{2 k_{3} m_{1} m_{2}}{m_{1} k_{2}-k_{1} m_{2}}
$$

If $C=0$ we can utilize Theorem 4 . Conditions which are necessary and sufficient for $G$ and $G K$ to be positive definite are found as

$$
\begin{aligned}
m_{1} m_{2}-g^{2} & >0, \\
m_{2} k_{1}+m_{1} k_{2} & >0, \\
\left(k_{3}^{2}+k_{1} k_{2}\right)\left(m_{1} m_{2}-g\right) & >0,
\end{aligned}
$$

assuming $m_{1}>0, m_{2}>0$. In the space of parameters $m_{1}, m_{2}, k_{1}, k_{2}, k_{3}$, these conditions define the exact region for which the equilibrium $(y, \dot{y})=(0,0)$ is stable for $C=0$. The above $G$ matrix may also be used with various nonzero forms for the operator $C$ to prove asymptotic stability, stability, or instability via Theorems 1, 2, and 3 . For example, since (3.5) implies $G$ and $G K$ are both positive definite, "proportional damping" of the form $C=c_{1} I+c_{2} K$ results in asymptotic stability by Theorem 1 if $\left(c_{1} c_{2} \geq 0\right.$, $\left.c_{1}+c_{2}>0\right)$ and instability by Theorem 3 if $\left(c_{1} c_{2} \geq 0, c_{1}+c_{2}<0\right)$, provided conditions (3.5) are satisfied.

Other forms for $C$ are easily considered by direct computation of $G C$. If the above theorems should happen to not apply for some given $C$ and the above $G$, one simply seeks a new $G$ such that $G$ and $G K$ are symmetric and $G C$ is non-negative. Usually one or more of the above theorems will then apply. For example, consider the general $C$ of (3.3) which may be both "nonproportional" and indefinite. The general form for $G$ such that both $G$ and $G K$ are symmetric is

$$
G=\left[\begin{array}{ll}
\alpha_{1} & g \\
g & \alpha_{2}
\end{array}\right], \quad g=\frac{\alpha_{2} m_{1} k_{3}+\alpha_{1} m_{2} k_{3}}{m_{1} k_{2}-m_{2} k_{1}}
$$

giving

$$
\begin{aligned}
G K & =\frac{1}{m_{1} m_{2}}\left[\begin{array}{ll}
\alpha_{1} m_{2} k_{1}+g m_{1} k_{3} & -\alpha_{1} m_{2} k_{3}+g m_{1} k_{2} \\
g m_{2} k_{1}+\alpha_{2} m_{1} k_{3} & -g m_{2} k_{3}+\alpha_{2} m_{1} k_{2}
\end{array}\right], \\
G C & =\frac{1}{m_{1} m_{2}}\left[\begin{array}{ll}
\alpha_{1} m_{2} c_{1}+g m_{1} c_{3} & \alpha_{1} m_{2} c_{2}+g m_{1} c_{4} \\
g m_{2} c_{1}+\alpha_{2} m_{1} c_{3} & g m_{2} c_{2}+\alpha_{2} m_{1} c_{4}
\end{array}\right] .
\end{aligned}
$$

The parameters $\alpha_{1}$ and $\alpha_{2}$ are still completely free. If we choose them such that $G C$ is non-negative,

$$
\frac{1}{m_{1} m_{2}}\left(\alpha_{1} m_{2} c_{1}+g m_{1} c_{3}\right) \geq 0
$$

$\frac{1}{m_{1} m_{2}}\left[4\left(\alpha_{1} m_{2} c_{1}+g m_{1} c_{3}\right)\left(g m_{2} c_{2}+\alpha_{2} m_{1} c_{4}\right)-\left(\alpha_{1} m_{2} c_{2}+g m_{1} c_{4}+g m_{2} c_{1}+\alpha_{2} m_{1} c_{3}\right)^{2}\right] \geq 0$ 
then Theorems 1, 2, or 3 may be used to predict asymptotic stability, stability, or instability, depending upon the definiteness or indefiniteness of $G$ and $G K$ for this choice of $\alpha_{1}$ and $\alpha_{2}$. It should be emphasized that if $\alpha_{1}$ and $\alpha_{2}$ are chosen to strictly satisfy (3.9), (3.10), we definitely have an answer since either Theorem 1 or Theorem 3 must then apply. This illustrates the fact that, in direct contrast to eigenvalue analysis, it is critical stability which is most difficult to determine when using a Liapunov approach.

4. Existence and properties of $G$. We can prove the existence of a family of operators $G$ having at least as many linearly-independent members as there are distinct eigenvalues of $K$. In order to do this we first construct a complex Hilbert space $\mathfrak{F}^{c},(u+i v) \in \mathfrak{K}^{c}$ iff $u, v \in \mathfrak{F}$, having the inner product $\left\langle u_{1}+i v_{1}, u_{2}+i v_{2}\right\rangle_{c}=\left\langle u_{1}, u_{2}\right\rangle+i\left\langle v_{1}, u_{2}\right\rangle-$ $i\left\langle u_{1}, v_{2}\right\rangle+\left\langle v_{1}, v_{2}\right\rangle$ for all $u_{1}, v_{1}, u_{2}, v_{2} \in \mathcal{F}$. We also temporarily extend the real operator $K$ to the complex domain $\mathcal{Y}^{c},\left(y_{1}+i y_{2}\right) \in \mathcal{Y}^{c}$ iff $y_{1}, y_{2} \in \mathcal{Y}$, and denote its adjoint by $K^{*}$ with complex domain of definition $z^{c}$. (Later we will also make use of the real linear manifold $z \triangleq z^{c} \cap \mathcal{K C}$.)

We now consider the associated eigenvalue problem

$$
K^{*} g=\lambda g, \quad g \in z^{c}
$$

and note that the eigenvalues $\lambda_{i}$ and eigenvectors $g_{i}$ either are real or occur in complex conjugate pairs since $K^{*}$ is a real operator. Define the operation

$$
G_{i} u \triangleq c_{i} g_{i}\left\langle g_{i}, u\right\rangle_{c}+\bar{c}_{i} \bar{g}_{i}\left\langle u, g_{i}\right\rangle_{c}, \quad u \in \mathfrak{F},
$$

where $(\bar{\cdot})$ denotes the complex conjugate of $(\cdot)$ and $c_{i}$ is an arbitrary complex constant. Since $\left\langle u_{1}, u_{2}\right\rangle_{c}=\overline{\left\langle u_{2}, u_{1}\right\rangle_{c}}$ we see that $G_{i}$ is a real symmetric operator defined on $\mathfrak{F}$. In addition,

$$
\begin{aligned}
G_{i} K y & =c_{i} g_{i}\left\langle g_{i}, K y\right\rangle_{c}+\bar{c}_{i} \bar{g}_{i}\left\langle K y, g_{i}\right\rangle_{c} \\
& =c_{i} g_{i}\left\langle K^{*} g_{i}, y\right\rangle_{c}+\bar{c}_{i} \bar{g}_{i}\left\langle y, K^{*} g_{i}\right\rangle_{c} \\
& =c_{i} \lambda_{i} g_{i}\left\langle g_{i}, y\right\rangle_{c}+\bar{\lambda}_{i} \bar{c}_{i} \bar{g}_{i}\left\langle y, g_{i}\right\rangle_{c}, \quad y \in \mathcal{Y} .
\end{aligned}
$$

Since $G_{i} K$ is also symmetric, $G_{i}$ satisfies (2.4).

Since $c_{i}$ is an arbitrary complex constant, each $G_{i}$ defined above actually involves two independent operators when $\lambda_{i}$ is complex. Therefore we have demonstrated the existence of at least as many linearly-independent operators $G_{i}$ satisfying (2.4) as there are distinct eigenvalues of $K$. This is clearly not a desirable way to find a family of operators $G$ satisfying (2.4), since eigenvalue analysis is what we are trying to avoid, and also the family (4.2) is parameterized in a way which is inconvenient for practical usage. Therefore we will consider some additional ways in which a family of operators satisfying (2.4) can be obtained, provided at least one member of the family is known.

Suppose some known operator $G_{0}$ satisfies (2.4). Then, since $G_{0}$ is symmetric,

$$
\begin{aligned}
\left\langle u_{1}, G_{0} K^{-1} u_{2}\right\rangle & =\left\langle G_{0} u_{1}, K^{-1} u_{2}\right\rangle \\
& =\left\langle G_{0} K K^{-1} u_{1}, K^{-1} u_{2}\right\rangle, \quad u_{1}, u_{2} \in \mathfrak{F},
\end{aligned}
$$

and since $\mathfrak{D}_{K}=\mathcal{Y}$ implies $\mathfrak{R}_{K^{-1}}=\mathcal{Y}$,

$$
\begin{aligned}
\left\langle u_{1}, G_{0} K^{-1} u_{2}\right\rangle & =\left\langle K^{-1} u_{1}, G_{0} K K^{-1} u_{2}\right\rangle \\
& =\left\langle G_{0} K^{-1} u_{1}, u_{2}\right\rangle, \quad u_{1}, u_{2} \in \mathcal{F}
\end{aligned}
$$


by the symmetry of $G_{0} K$. Therefore $G_{0} K^{-1}$ also satisfies (2.4). Since this process can be continued we see that we can generate an entire family of operators satisfying (2.4) by

$$
G=G_{0}, G_{0} K^{-1}, G_{0} K^{-2}, \cdots .
$$

It can be similarly shown that families could be developed as

$$
G=G_{0}, K^{*-1} G_{0}, K^{*-2} G_{0}, \cdots
$$

or

$$
G=G_{0}(K-\lambda I)^{-1}
$$

or

$$
G=\left(K^{*}-\lambda I\right)^{-1} G_{0},
$$

where $\lambda$ is a real number not in the spectrum of $K$ or $K^{*}$.

The last two forms are parameterized by $\lambda$ in a way which is very useful in applying the Liapunov approach, in that $\lambda$ can often be chosen such that $G C$ is definite on $\mathcal{Y}$, or at least semidefinite on $\mathcal{Y}$, for the operator $C$ which is of interest. Note that $C$ is not involved in determining any of the above families, but does determine whether or not any particular operator $G$ will generate a useful Liapunov functional via (2.2) or (2.5).

At present the most general method found for generating families which satisfy (2.4), given one $G_{0}$ which does satisfy (2.4), is via

$$
G=G_{0}\left[P_{1}(K)\right]^{-1}, \quad G_{0}\left[P_{2}(K)\right]^{-1}, \cdots
$$

or

$$
G=\left[P_{1}\left(K^{*}\right)\right]^{-1} G_{0}, \quad\left[P_{2}\left(K^{*}\right)\right]^{-1} G_{0}, \cdots
$$

where the $P_{i}$ are arbitrary real polynomials for which the inverses exist.

5. Distributed-parameter systems. In the following sections we will consider distributed-parameter examples involving a mass continuously distributed over a bounded region $\Omega=(0,1)$, where $K$ is a differential operator of order $2 n$ and $C$ is also a differential operator of order $\leq 2 n$. A natural choice for $\mathcal{H C}$ is the space $\mathfrak{L}_{2}(0,1)$, and consequently $\mathcal{Y}$ is chosen as the set of functions in the Sobolev space $\mathcal{F}_{2}^{(2 n)}(0,1)$ which satisfy $n$ linear homogeneous boundary conditions at each boundary point of $\Omega^{\dagger} . \mathcal{F}_{2}^{(2 n)}(0,1)$ is the linear space of all summable functions $u(x)$ having on the open set $(0,1)$ all generalized derivatives of order $\leq 2 n$ summable to the power 2 . Thus $\mathfrak{F C}_{2}^{(2 n)}(0,1) \subset \mathcal{F C}_{2}^{(0)}(0,1)=\mathfrak{L}_{2}(0,1)$ and $\mathcal{Y}$ is a linear manifold in $\mathcal{H C}$. The adjoint operator $K^{*}$ and its domain $z$ are similarly defined. Our objective will be to determine operators $G$ satisfying (2.4) such that $V$ of (2.2), or $U$ of (2.5), or some linear combination of $V$ and $U$, is a useful Liapunov functional for the system considered.

From (2.4) we see that $G$ is to be symmetric and defined on $\mathfrak{F}=\mathfrak{L}_{2}(0,1)$, and this implies that $G$ cannot be a differential operator of order larger than zero. This is not very informative and we must have a clear idea of the form of $G$ before we can use (2.4) to devise a specific technique for finding $G$. For this purpose we will make an assumption on the form of $G$, based on what we prefer rather than what we require. Noting that we

† The boundary conditions will involve $y$ and its first $2 n-1$ derivatives, and the Sobolev embedding theorem implies that $y \in \mathbb{e}^{(2 n-1)}(0,1)$ if $y \in \mathcal{F}_{2}{ }^{(2 n)}(0,1)$. 
plan to use and manipulate $G$ in the functionals (2.2), (2.3), (2.5), (2.6), we will here make the assumption that $G$ has an inverse which is an ordinary differential operator of order $\leq 2 n$. This assumption is not necessary, but under it we can present a simple technique that works for all symmetric $K$ and at least some nonsymmetric $K$.

Suppose the differential operator $(K-\lambda I)$, where $\lambda$ is a real constant not in the spectrum of $K$, may be factored into two real linear differential operators

$$
K-\lambda I=B A
$$

such that

(i) $A$ is symmetric, $D_{A}=Y$,

(ii) $B$ is symmetric, $D_{B}=\Omega_{A}$,

(iii) $B^{-1}$ exists, $D_{B^{-1}}=\mathscr{R}_{B}$.

If this can be done, note that $D_{B^{-1}}=R_{B}=R_{K}=\mathfrak{F}$ and define

$$
G=B^{-1}
$$

which is an integral operator with a symmetric kernel or an ordinary function. Also

$$
G K y=B^{-1}(B A+\lambda I) y=A y+\lambda B^{-1} y
$$

which is symmetric on $\mathcal{Y}$. This implies $G=B^{-1}$ satisfies (2.4).

We note that the above factorization is always possible when $K$ is symmetric. Choosing $A=K-\lambda I, B=I$, we get $G=I$ and so produce the energy functional for $V$ by (2.2). We can also choose $A=I, B=K-\lambda I$, and so produce $G=(K-\lambda I)^{-1}$ which is actually a family parameterized by $\lambda$. This latter family may produce stability results for forms of the operator $C$ with which the energy functional fails. Other simple choices are also sometimes possible.

For nonsymmetric $K$, the above factorization is more difficult since $A$ and $B$ must generally be variable-parameter, even for a constant-parameter $K$. Whether or not the above factorization is always possible is still an open question, but we will show by example that it is sometimes possible.

Let us now consider the forms $V$ and $\dot{V}$ :

$$
\begin{gathered}
V=\langle\dot{y}, G \dot{y}\rangle+\langle y, G K y\rangle \\
\dot{V}=-2\langle\dot{y}, G C \dot{y}\rangle, \quad(y, \dot{y}) \in \mathcal{y} \times \mathcal{Y} .
\end{gathered}
$$

The operator $G=B^{-1}$ may be either a simple multiplying function or an integral operator. If it is an integral operator we may have some practical difficulties when trying to determine the definiteness properties of $V$ and $\dot{V}$. We therefore suggest the following change. Define a domain $\mathscr{W}=G(\mathcal{Y})$, note that $G=B^{-1}$ provides a one-to-one mapping of $\mathcal{Y}$ onto $\mathscr{W}$, and consider the functionals $V$ and $\dot{V}$ in the form

$$
\begin{aligned}
& V=\langle\dot{w}, B \dot{w}\rangle+\langle w, K B w\rangle, \\
& \dot{V}=-2\langle\dot{w}, C B \dot{w}\rangle, \quad(w, \dot{w}) \in w \times w .
\end{aligned}
$$

These functionals involve only the differential operators $B, C, K$, and are therefore amenable to a variety of techniques for determining definiteness properties, such as variational calculus. In addition these functionals do not require the evaluation of the operator $B^{-1}$. 
In application it will be convenient to note that

$$
W=G(\mathcal{Y})=\{u \mid u \in z, B u \in \mathcal{Y}\},
$$

where $z$ is the domain of the adjoint operator $K^{*}$. This can be shown by noting that

$$
G(\mathcal{Y}) \triangleq\left\{u \mid u \in D_{B}, B u \in \mathcal{Y}\right\}
$$

while (2.4) implies $G K y=K^{*} G^{*} y=K^{*} G y$ for all $y \in \mathcal{Y}$ and therefore $G(\mathcal{Y}) \subset z$. Consequently (5.10) implies

$$
G(\mathcal{Y}) \subset\{u \mid u \in z, B u \in \mathcal{Y}\} .
$$

On the other hand, $B^{*}=B$ and $D_{B^{*}}=D_{B}$, since $B$ is symmetric and $\Omega_{B}=\mathcal{F}$ by (5.2). Noting that the adjoint of (5.1), $K^{*}-\lambda I=A^{*} B^{*}$, is defined on $z$, we see that $\mathscr{D}_{B}=$ $D_{B^{*}} \supset$ z. By again using (5.10) we now have

$$
G(\mathcal{Y}) \supset\{u \mid u \in z, B u \in \mathcal{Y}\} \text {, }
$$

and (5.9) follows from (5.11) and (5.12).

At this point something should be said about the meaning of stability. The general definitions of stability, instability, and asymptotic stability are completely analogous for general and lumped-parameter systems, the only distinction being the norm-dependence of these concepts for distributed-parameter systems [7]. For the sake of simplifying and unifying the stability conclusions reached for the examples of the following section, no mention will be made in these examples of the norm or norms being used to draw these conclusions. In fact we will proceed almost as though Theorems 1-4 of Sec. 3 carried over directly to distributed-parameter systems. In a sense they do, provided the proper norms are used.

Consider, for example, Theorem 2 of Sec. 3. Suppose the technique of the present section has produced a $G=B^{-1}$, where $B$ satisfies (5.2), and the conditions of Theorem 2 hold in the sense that $B$ and $K B$ are positive on $\mathscr{W}=B^{-1}(\mathcal{Y})$ with respect to the $\mathcal{L}_{2}(0,1)$ norm of $\Re$, and $C B$ is non-negative on $W$. We have by (5.7) and (5.8)

$$
\begin{aligned}
& V(w, \dot{w})=\langle\dot{w}, B \dot{w}\rangle+\langle w, K B w\rangle, \\
& \dot{V}(w, \dot{w}) \leq 0, \quad(w, \dot{w}) \in w \times w,
\end{aligned}
$$

where $K$ is a differential operator of order $2 n$ and $B$ is a differential operator of order $2 m \leq 2 n$. Stability can be concluded relative to any norm $\|(w, \dot{w})\|_{s}$ which is equivalent to $(V(w, \dot{w}))^{1 / 2}$; i.e., there exist two positive constants $c_{1}, c_{2}$, such that

$$
c_{1} \|\left(w, \dot{w}\left\|_{S}^{2} \geq V(w, \dot{w}) \geq c_{2}\right\|(w, \dot{w}) \|_{S}^{2}\right.
$$

for all $(w, \dot{w}) \in \mathscr{W} \times \mathfrak{W}$. The value of $\left\|\left(B^{-1} y, B^{-1} \dot{y}\right)\right\|_{s}$, along any solution of $(2.1)$, is then bounded above in terms of its initial value. In many cases an appropriate stability norm is the norm of $\mathfrak{F C}_{2}^{(n+m)}(0,1) \times \mathfrak{F C}_{2}^{(m)}(0,1)$.

Now suppose that Theorem 1 is satisfied in the sense that, in addition to the above, $C B$ is positive on $W$ with respect to the $\mathscr{L}_{2}(0,1)$ norm of $\mathfrak{F}$. Clearly the equilibrium is stable in the above sense, but in what way is the stability asymptotic as concluded by Theorem 1? LaSalle's invariance principle [9] was employed in the proof of Theorem 1 using only the functional $V$ of (2.2) [6]. However, the various versions of this principle for general systems are quite complicated $[7,10,11]$, so let us avoid its use by the following 
device. Substitute $G K^{-1}$ for $G$ in $U$ and $\dot{U}$ of (2.5) and (2.6), add the results to $V$ and $\dot{V}$ of (2.2) and (2.3), and substitute $B w$ and $B \dot{w}$ for $y$ and $\dot{y}$, thereby forming new functionals

$$
\begin{aligned}
\underline{V}(w, \dot{w}) & =\langle\dot{w}, B w\rangle+\langle w, K B w\rangle \\
& +\left\langle K^{-1}(B \dot{w}+C B w), B^{-1}(B \dot{w}+C B w)\right\rangle+\langle w, B w\rangle, \\
\dot{V}(w, \dot{w}) & =-2\langle C B \dot{w}, \dot{w}\rangle-2\langle C B w, w\rangle, \quad(w, \dot{w}) \in w \times w .
\end{aligned}
$$

Quasi-asymptotic stability can be concluded relative to any norm $\|(w, w)\|_{O_{A S}}$ which bounds $(-\underline{\dot{V}}(w, \dot{w}))^{1 / 2}$ from below; i.e., there exists a positive constant $c_{3}$ such that

$$
-\dot{V}(w, \dot{w}) \geq c_{3}\|(w, \dot{w})\|_{O A S}^{2}
$$

for all $(w, \dot{w}) \in \mathscr{W} \times \mathfrak{W}$. The norm of $\mathfrak{L}_{2}(0,1)$ is certainly a possible choice for $\|(w, w)\|_{Q A S}$ since $C B$ was assumed positive with respect to this norm, but a stronger choice can often be made. The value of $\left\|\left(B^{-1} y, B^{-1} \dot{y}\right)\right\|_{Q A S}$, along any solution of (2.1), approaches zero as $t \rightarrow \infty$. In at least this restricted sense of stability with respect to $\|(w, \dot{w})\|_{s}$ and quasi-asymptotic stability with respect to $\|(w, \dot{w})\|_{Q A S}$, the equilibrium $(y, \dot{y})=$ $(0,0)$ is asymptotically stable.

There are many other approaches that may be used in particular cases. For example, if $C$ is a constant we may directly add $V$ to $U, \dot{V}$ to $\dot{U}$, substitute $B w$ for $y$ and $B \dot{w}$ for $\dot{y}$, and form the functionals

$$
\begin{aligned}
& \underline{V}(w, \dot{w})=\langle\dot{w}, B \dot{w}\rangle+\left\langle(B \dot{w}+C B w), B^{-1}(B \dot{w}+C B w)\right\rangle+2\langle w, K B w\rangle, \\
& \dot{V}(w, \dot{w})=-2\langle C B \dot{w}, \dot{w}\rangle-2\langle K B w, C w\rangle, \quad(w, \dot{w}) \in w \times w .
\end{aligned}
$$

If $B$ and $K B$ are positive with respect to the $\mathscr{L}_{2}(0,1)$ norm and $(V(w, \dot{w}))^{1 / 2}$ is equivalent to $\|(w, \dot{w})\|_{s}$, then $(-\dot{V}(w, \dot{w}))^{1 / 2}$ is also usually equivalent to $\|(w, \dot{w})\|_{s}$ for $C>0$. Therefore we may conclude true asymptotic stability relative to this norm, which applies to both boundedness and limit behavior. If the constant $C=0(C<0)$ stability (instability) is proved relative to this same norm.

Generally each problem and each Liapunov functional should be investigated on its own merits to determine the precise meaning of the stability conclusions drawn. At present there appear to be few simple rules of practical value, and the reader is referred to more basic work along these lines $[8,10,11,12]$. Here we are concerned with generating Liapunov functionals rather than demonstrating rigorous methods of usage, and so stability results for the following examples will be stated imprecisely.

There is another and much more subtle question which may occur to the reader: do the defining equations of the following examples actually define dynamical systems on $\mathcal{Y} \times \mathcal{Y}=\mathfrak{F}_{2}^{(2 n)}(0,1) \times \mathcal{F C}_{2}^{(2 n)}(0,1)$, as was assumed for (1.1)? This is a question which it is neither necessary nor possible to answer here, given our present objective, and again more basic work should be consulted [7, 10,11]. Here we shall simply be optimistic and assume that in each of the following examples there exists a general solution having the semigroup property and providing a continuous mapping of $\left[t_{0}, \infty\right) \times$ $\mathcal{Y} \times \mathcal{y}$ into $\mathcal{Y} \times \mathcal{y}$. 
6. Example: pin-ended Euler column. The pin-ended Euler column is dynamically described by

$$
\rho(x) \ddot{y}(t)+\hat{C} \dot{y}(t)+\left(\partial^{4}+p \partial^{2}\right) y(t)=0
$$

with boundary conditions $y=\partial^{2} y=0$ at $x=0,1$, where $\rho(x)>0$ for all $x \in[0,1]$, the constant $p \neq m^{2} \pi^{2}, m=1,2, \cdots$, and $\hat{C}$ is a differential operator of order $\leq 4$.

This system is seen to be given in the form (1.1) and both $\hat{M}$ and $\hat{K}$ are symmetric. Therefore there exists an energy functional

$$
2 E=\int_{0}^{1} \rho \dot{y}^{2} d x+\int_{0}^{1} y \cdot\left(\partial^{4}+p \partial^{2}\right) y d x
$$

having the time derivative

$$
2 \dot{E}=-2 \int_{0}^{1} \dot{y} \cdot \hat{C} \dot{y} d x .
$$

The functional $2 E$ is useful as a Liapunov functional if $\hat{C}$ is definite on $\mathcal{Y}$, and may be useful if $\hat{C}$ is only semidefinite on $\mathcal{Y}$. It will not be useful if $\hat{C}$ is indefinite on $\mathcal{Y}$. In any case, we may wish to have some other candidates for Liapunov functionals and so we place the system in the form (2.1) where

$$
\begin{aligned}
& \ddot{y}(t)+C \dot{y}(t)+K y(t)=0, \quad(y, \dot{y}) \in \mathcal{Y} \times \mathcal{Y}, \\
& K=\frac{1}{\rho}\left(\partial^{4}+p \partial^{2}\right), \quad \mathcal{Y}=\left\{\begin{array}{l|l}
u \in \mathcal{K}_{2}^{(4)}(0,1) & \begin{array}{l}
u=\partial^{2} u=0 @ x=0 \\
u=\partial^{2} u=0 @ x=1
\end{array}
\end{array}\right\} \\
& K^{*}=\left(\partial^{4}+p \partial^{2}\right)\left(\frac{\dot{q}}{\rho}\right), \quad z=\left\{\begin{array}{l|l}
u \in \mathcal{F C}_{2}^{(4)}(0,1) & \begin{array}{l}
u=\partial^{2}\left(\frac{u}{\rho}\right)=0 @ x=0 \\
u=\partial^{2}\left(\frac{u}{\rho}\right)=0 @ x=1
\end{array}
\end{array}\right\} \\
& C=(1 / \rho) \hat{C}, \quad\left\langle u_{1}, u_{2}\right\rangle \triangleq \int_{0}^{1} u_{1}(x) u_{2}(x) d x, \quad u_{1}, u_{2} \in \mathcal{H C}=\mathscr{L}_{2}(0,1) .
\end{aligned}
$$

Now we consider

$$
(K-\lambda I)(\cdot)=(1 / \rho)\left(\partial^{4}+p \partial^{2}-\lambda \rho\right)
$$

and note there are several ways to perform the factorization (5.1) while satisfying (5.2):

i) $A=\left(\partial^{4}+p \partial^{2}-\lambda \rho\right), B=1 / \rho$,

ii) $A=\rho, B(\cdot)=(1 / \rho)\left(\partial^{4}+p \partial^{2}-\lambda \rho\right)(\cdot / \rho)$,

iii) $A=\left(\partial^{2}+p-\beta\right), B=(1 / \rho)\left(\partial^{2}+\beta\right), \beta^{2}-p \beta=\rho \lambda$,

where (i) and (ii) are valid for all $\rho(x)(\rho(x)>0$ for all $x \in[0,1])$ and (iii) is valid only for constant $\rho>0$.

First consider (i), for which $G=B^{-1}=\rho(x), W_{1} \triangleq G(Y)=z$. Then by (5.7) and (5.8)

$$
\begin{aligned}
& V_{1}=\int_{0}^{1} \frac{1}{\rho} \dot{w}^{2} d x+\int_{0}^{1} \frac{w}{\rho} \cdot\left(\partial^{4}+p \partial^{2}\right)\left(\frac{w}{\rho}\right) d x, \\
& \dot{V}_{1}=-2 \int_{0}^{1} \frac{\dot{w}}{\rho} \cdot \hat{C}\left(\frac{\dot{w}}{\rho}\right) d x, \quad(w, \dot{w}) \in z \times z,
\end{aligned}
$$


or, equivalently, by (5.5) and (5.6),

$$
\begin{aligned}
& V_{1}=\int_{0}^{1} \rho \dot{y}^{2} d x+\int_{0}^{1} y \cdot\left(\partial^{4}+p \partial^{2}\right) y d x, \\
& \dot{V}_{1}=-2 \int_{0}^{1} \dot{y} \cdot \hat{C} \dot{y} d x, \quad(y, \dot{y}) \in \mathcal{Y} \times \mathcal{Y} .
\end{aligned}
$$

In the latter form it is apparent that $V_{1}$ is just the energy functional $2 E$ for the system (6.1). It is not the energy functional for the system (6.4) from which it was derived, however. In fact, (6.4) has no energy functional since $K$ is not symmetric. We see that $V_{1}$ is (may be) a Liapunov functional for all definite (semidefinite) operators $\hat{C}$. If $\hat{C}$ is a positive operator and $p<\pi^{2}\left(p>\pi^{2}\right)$ the equilibrium $(y, \dot{y})=(0,0)$ is asymptotically stable (unstable). If $\hat{C}$ is a negative operator the equilibrium is unstable for all $p$. If $\hat{C}$ is non-negative, and $p<\pi^{2}$, the equilibrium is at least stable. If $\hat{C}$ is indefinite, no result is obtained from $V_{1}$.

In contrast to the preceding, case (ii) provides a family of operators $G=\rho\left(\partial^{4}+\right.$ $\left.p \partial^{2}-\lambda \rho\right)^{-1} \rho$, and

$$
\begin{aligned}
& W_{2} \triangleq G(Y)=\left\{u \mid u \in z,(1 / \rho)\left(\partial^{4}+p \partial^{2}-\lambda \rho\right)(u / \rho) \in \mathcal{Y}\right\}, \\
& V_{2}=\int_{0}^{1} \frac{\dot{w}}{\rho} \cdot\left(\partial^{4}+p \partial^{2}-\lambda \rho\right)\left(\frac{\dot{w}}{\rho}\right) d x+\int_{0}^{1} \frac{w}{\rho} \cdot\left(\partial^{4}+p \partial^{2}\right) \frac{1}{\rho}\left(\partial^{4}+p \partial^{2}-\lambda \rho\right)\left(\frac{w}{\rho}\right) d x, \\
& \dot{V}_{2}=-2 \int_{0}^{1} \frac{\dot{w}}{\rho} \cdot \hat{C} \frac{1}{\rho}\left(\partial^{4}+p \partial^{2}-\lambda \rho\right)\left(\frac{\dot{w}}{\rho}\right) d x, \quad(w, \dot{w}) \in W_{2} \times W_{2} .
\end{aligned}
$$

Given any operator $\hat{C}$, we may here attempt to choose $\lambda$ in such a way that $(1 / \rho)$ $\cdot \hat{C}(1 / \rho)\left(\partial^{4}+p \partial^{2}-\lambda \rho\right)(\cdot / \rho)$ is a definite or semidefinite operator on $W_{2}$. For example, suppose $\hat{C}=\left(\partial^{4}+p \partial^{2}+c \rho\right)$ and choose $\lambda=-c . V_{2}$ is then a Liapunov functional for any $p$ and any $\rho(x)$, regardless of whether $\hat{C}$ is definite or indefinite. This form for $\hat{C}$ is seen to be "proportional", but this is not meant to exclude any other form for $\hat{C}$ such that $(1 / \rho) \hat{C}(1 / \rho)\left(\partial^{4}+p \partial^{2}-\lambda \rho\right)(\cdot / \rho)$ is at least semidefinite on $W_{2}$ for some choice of $\lambda$.

We now consider case (iii) where $G=\rho\left(\partial^{2}+\beta\right)^{-1}$ and $\rho$ and $\beta$ are constants:

$$
\begin{aligned}
& \mathscr{W}_{3}=\left\{u \mid u \in z,\left(\partial^{2}+\beta\right)(u / \rho) \in \mathcal{Y}\right\}, \\
& V_{\mathbf{3}}=\int_{0}^{1} \dot{w} \cdot\left(\partial^{2}+\beta\right)\left(\frac{\dot{w}}{\rho}\right) d x+\int_{0}^{1} \frac{w}{\rho} \cdot\left(\partial^{4}+p \partial^{2}\right)\left(\partial^{2}+\beta\right)\left(\frac{w}{\rho}\right) d x, \\
& \dot{V}_{3}=-2 \int_{0}^{1} \frac{\dot{w}}{\rho} \cdot \hat{C}\left(\partial^{2}+\beta\right)\left(\frac{\dot{w}}{\rho}\right) d x, \quad(w, \dot{w}) \in W_{3} \times W_{3} .
\end{aligned}
$$

The functional $V_{3}$ may be used in the same manner as $V_{2}$, and has a certain practical advantage since only operators of up to sixth (rather than eighth) order are involved. We choose $\beta$ such that $(1 / \rho) \hat{C}\left(\partial^{2}+\beta\right)(\cdot / \rho)$ is definite or semidefinite on $W_{3}$. As an obvious example, consider $\hat{C}=\left(\partial^{2}+\alpha\right)$, and choose $\beta=\alpha$. Note that this $\hat{C}$ is neither "proportional" nor, in general, definite.

We note that we have not had actually to evaluate the operator $G$. This is an attribute of the technique described in Sec. 5 . We must, of course, be sure that $G$ exists. It was assumed in the preceding that $\lambda$ was such that $G=B^{-1}$ did indeed exist. 
7. Example: circulatory string. Consider the motion of a string of variable density, subjected to a circulatory loading proportional to the slope and another loading dependent upon velocity. The system is described by

$$
\rho(x) \ddot{y}(t)+\hat{C} \dot{y}(t)-\left(\partial^{2}+p \partial\right) y(t)=0
$$

with boundary conditions $y=0$ at $x=0,1$ where $\rho(x)>0$ for all $x \in[0,1]$ and $p$ is a constant. $\hat{C}$ is a differential operator of order $\leq 2$. In the notation of Sec. 2 ,

$$
\begin{array}{r}
K=(-1 / \rho)\left(\partial^{2}+p \partial\right), \quad K^{*}=-\left(\partial^{2}-p \partial\right)(\cdot / \rho), \quad C=(1 / \rho) \hat{C}, \quad \mathcal{F}=\mathscr{L}_{2}(0,1), \\
\mathcal{Y}=z=\left\{u \in \mathcal{F C}_{2}^{(2)}(0,1) \mid u=0 @ x=0, u=0 @ x=1\right\}, \quad \text { (7.2) }
\end{array}
$$

and

$$
K-\lambda I=(-1 / \rho)\left(\partial^{2}+p \partial\right)-\lambda=(-1 / \rho) \partial e^{-p x} \partial e^{p x}-\lambda .
$$

We may therefore let

(i) $A=\rho e^{p x}, B(\cdot)=(-1 / \rho)\left(\partial e^{-p x} \partial+\lambda e^{-p x}\right)(\cdot / \rho)$

(ii) $A(\cdot)=-\left(e^{p x} \partial e^{-p x} \partial e^{p x}+\lambda \rho e^{p x}\right)(\cdot), B=\left(e^{-p x} / \rho\right)$,

and find for case (i) via (5.7), (5.8),

$$
\begin{aligned}
& W_{1}=\left\{u \mid u \in z,\left((-1 / \rho) \partial e^{-p x} \partial-\lambda e^{-p x}\right)(u / \rho) \in \mathcal{Y}\right\}, \\
& V_{1}=-\int_{0}^{1} \dot{w} \cdot\left(\frac{1}{\rho} \partial e^{-p x} \partial+\frac{\lambda e^{-p x}}{\rho}\right)\left(\frac{\dot{w}}{\rho}\right) d x+\int_{0}^{1} \frac{w}{\rho} \cdot\left(\partial^{2}+p \partial\right)\left(\frac{1}{\rho} \partial e^{-p x} \partial+\frac{\lambda e^{-p x}}{\rho}\right)\left(\frac{w}{\rho}\right) d x, \\
& \dot{V}_{1}=2 \int_{0}^{1} \frac{\dot{w}}{\rho} \cdot \hat{C}\left(\frac{1}{\rho} \partial e^{-p x} \partial+\frac{\lambda e^{-p x}}{\rho}\right) \dot{w} d x, \quad(w, \dot{w}) \in W_{1} \times W_{1} .
\end{aligned}
$$

Again we have a parameterized family of $V$ functionals, the constant $\lambda$ to be chosen, upon specification of $\hat{C}$, so as to make $(1 / \rho) \hat{C}\left((1 / \rho) \partial e^{-p x} \partial+\left(\lambda e^{-p x} / \rho\right)\right)$ at least semidefinite on $W_{1}$.

For case (ii), we obtain only one functional $V_{2}$ by using $G=B^{-1}=\rho e^{p x}$ in (5.5):

$$
V_{2}=\int_{0}^{1} \rho e^{p x} \dot{y}^{2} d x-\int_{0}^{1} y e^{p x} \cdot\left(\partial^{2}+p \partial\right) y d x
$$

and

$$
\dot{V}_{2}=-2 \int_{0}^{1} \dot{y} e^{p x} \cdot \hat{C} \dot{y} d x, \quad(y, \dot{y}) \in \mathcal{Y} \times \mathcal{Y} .
$$

$V_{2}$ is also a Liapunov functional for certain forms of $\hat{C}$. For constant $\hat{C}=2 \xi, V_{2}$ and $\dot{V}_{2}$ imply asymptotic stability for all $\xi>0$, critical stability for $\xi=0$, and instability for $\xi<0$, these results being completely independent of $p$ and $\rho(x)(\rho(x)>0$ for all $x \in[0,1])$. This simple case is mentioned because Plaut and Infante [13] have considered this problem with constant $\rho$ and a $p(t)$ which is a stationary ergodic stochastic process and obtained a much more restrictive result. Their result is not improved for deterministic $p$, which by stationarity must be constant. The sharpness of the results obtained here is due to the fact that functionals of the form (2.2) and (2.5) are "perfect" Liapunov functionals, in the sense that they become integrals of the motion for $C=0$.

8. Example: column with circulatory loading. Consider a uniform column which is pinned at one end, restricted to zero slope at the other, and subjected to a uniform. 
tangential compressive loading $p>0$ which remains tangential regardless of the deformation. The system is described by

$$
\ddot{y}(t)+C \dot{y}(t)+K y(t)=0, \quad(y, \dot{y}) \in \mathcal{Y} \times \mathcal{Y}
$$

where $C$ is a differential operator of order $\leq 4$ and

$$
\begin{aligned}
& K=\left(\partial^{4}+p x \partial^{2}\right), \quad K^{*}=\left(\partial^{4}+p \partial^{2} x\right), \quad \mathfrak{H}=\mathfrak{L}_{2}(0,1), \\
& \mathcal{Y}=\left\{\begin{array}{ll}
u \in \mathcal{F C}_{2}^{(4)}(0,1) \mid \begin{array}{r}
u=\partial^{2} u=0 @ x=0 \\
\partial u=\partial^{3} u=0 @ x=1
\end{array}
\end{array}\right\}, \\
& z=\left\{u \in \mathcal{H}_{2}^{(4)}(0,1) \mid \begin{array}{c}
u=\partial^{2} u=0 @ x=0 \\
\partial u=\left(\partial^{3}+p\right) u=0 @ x=1
\end{array}\right\} .
\end{aligned}
$$

The operator $(K-\lambda I)$ may be factored for $\lambda=0$ as

$$
K=B A, \quad A=-\partial^{2}, \quad B=-\left(\partial^{2}+p x\right)
$$

and (5.7) and (5.8) become

$$
\begin{aligned}
& V=-\int_{0}^{1} \dot{w} \cdot\left(\partial^{2}+p x\right) \dot{w} d x-\int_{0}^{1} w \cdot\left(\partial^{2}+p x\right)\left(\partial^{2}\right)\left(\partial^{2}+p x\right) w d x, \\
& \dot{V}=2 \int_{0}^{1} \dot{w} \cdot C\left(\partial^{2}+p x\right) \dot{w} d x, \quad(w, \dot{w}) \in W \times W
\end{aligned}
$$

where

$$
\begin{aligned}
\mathscr{W} & =\{u \mid u \in z, B u \in \mathcal{Y}\}, \\
& =\left\{\begin{array}{l|l}
u \in \mathfrak{K}_{2}^{(\theta)}(0,1) & \begin{array}{l}
u=\partial^{2} u=\left(\partial^{4}+2 p \partial\right) u=0 @ x=0 \\
\partial u=\left(\partial^{3}+p\right) u=\left(\partial^{5}+p \partial^{3} u+3 p \partial^{2}\right) u=0 @ x=1
\end{array}
\end{array}\right\} .
\end{aligned}
$$

Integrating (8.4) by parts, we see that

$$
V=\int_{0}^{1} \dot{w} \cdot B \dot{w} d x+\int_{0}^{1} y \cdot A y d x, \quad \dot{w} \in W, \quad y \in \mathcal{Y} .
$$

$A$ is a positive operator on $Y$ and $B$ is positive (indefinite) on $W$ for $p<p_{c r} .\left(p>p_{c r}\right.$ ), where $p_{\text {cr. }}$ is the lowest value of $p$ such that $B w=0$ has a nonzero solution $w \in$ w. Therefore we conclude stability (asymptotic stability) of the equilibrium for any operator $C$ such that $C B$ is non-negative (positive) on $W$ and $p<p_{c r}$. We conclude instability for any operator $C$ such that $C B$ is definite on $W$ and $p>p_{c r}$. . For example, suppose the term $C \dot{y}$ in (8.1) is due to viscous damping, $C=2 \xi>0$. Then (8.5) becomes

$$
\dot{V}=-4 \xi \int_{0}^{1} \dot{w} \cdot B \dot{w} d x
$$

and asymptotic stability results for any $p<p_{c r}$. and instability occurs for $p>p_{c r}$.

If $C$ should be such that $C B$ is indefinite on $W$, many other functionals $V$ can be constructed by deriving new operators $G$ from $G_{0}=B^{-1}$, using the method explained in Sec. 4, until a $G$ is found such that $G C$ is not indefinite on $Y$. The functional $V$ of (8.4) is believed to be the first Liapunov functional ever found for this problem. 
9. Comments. An approach has been described for the generation of a set (or sets) of functionals suitable as simple candidates for a Liapunov functional for linear elastic dynamic systems. Basically the approach is to search for a family (or families) of linear operators $G$ satisfying (2.4). In Sec. 4 such operators were shown to exist for the general problem. The determination of these operators is sometimes nearly trivial as in Sec. 3 for lumped-parameter systems, and also for partial differential equations when $K$ is symmetric, using the technique described in Sec. 5. For many circulatory partial differential equations, however, the technique of Sec. 5 is far from trivial and in fact may not be workable. Certainly more work is needed on means of systematically satisfying (2.4) for distributed-parameter systems.

The present objective, however, was not the development of specific Liapunov functionals for specific problems, but rather the presentation of a general approach for general linear elastic dynamic systems defined on a Hilbert space which provides a desirable alternative to eigenvalue analysis. Such an approach has not only been developed but, through specific examples, it has been shown to be both feasible and desirable to use such an approach. Even for discrete systems we note that we search only for $n \times n$ matrices $G$, not for $2 n \times 2 n$ matrices as the general Liapunov equation requires [1]. We note also that at no point do we lose sight of the original operators, $C$ and $K$, involved in the problem and can detect throughout the effect of any changes or inaccuracies in these operators.

It should also be noted that when using the functional $V$ of (2.2), the operator $C$ need not actually be linear or time-independent. The conditions on $G$ in no way involve $C$, and the functional $V$ (but not $U$ of (2.5)) is also independent of $C$. That is, even for a nonlinear or time-dependent operator $C, \dot{V}$ of (2.3) is still the derivative of $V$ and the family of operators $G$ is still found in the same way. Of course, $C$ plays a decisive role in determining which member of this family one should use.

One general advantage of any Liapunov approach over eigenvalue analysis should also be emphasized. A Liapunov functional obtained on the basis of a given system model is not limited in usefulness to that particular model; it can be used with any system and, in particular, with more accurate descriptions of the modeled system. This allows not only the determination of a domain of permissible variations in some parameter space but also, in the case of nonlinearity in the modeled system, the determination of a "domain of attraction" in the initial state space. Therefore, despite the mathematical difficulties which we only touched upon here, it seems highly desirable to extend the simple usage of the Liapunov approach from discrete to distributed-parameter systems.

Acknowledgement. I wish to thank E. F. Infante of Brown University and A. Wouk of Northwestern University for their interest and comments during the course of this work.

\section{REFERENCES}

[1] W. Hahn, Theory and application of Liapunov's direct method, Prentice-Hall, 1963

[2] G. Hermann, Stability of equilibrium of elastic systems subjected to nonconservative forces, Appl. Mech. Rev. 20, 103-108 (1967)

[3] S. Nemat-Nasser and G. Hermann, Some general considerations concerning the destabilizing effect in nonconservative systems, ZAMP 17, 305-313 (1966)

[4] C. Urbano, The effect of damping on the dynamic stability of nonconservative systems, Meccanica 3, 131-139 (1968)

[5] V. I. Zubov, Methods of A. M. Lyapunov and their application, Noordhoff, 1964 
[6] J. A. Walker, On the stability of linear discrete dynamic systems, J. Appl. Mech. 37, 271-275 (1970) [7] J. K. Hale and E. F. Infante, Extended dynamical systems and stability theory, Proc. Nat. Acad. Sci. 58, 405-409 (1967)

[8] G. R. Buis, W. G. Vogt and M. M. Eisen, Lyapunov stability for partial differential equations, NASA Contractor Report 1100, June 1968

[9] J. P. LaSalle, Stability theory for ordinary differential equations, J. Diff. Eqs. 4, 57-65 (1968)

[10] J. K. Hale, Dynamical systems and stability, J. Math. Anal. Appl. 26, 39-59 (1969)

[11] M. Slemrod, Asymptotic behavior of a class of abstract dynamical systems, J. Diff. Eqs. 7, 584-600 (1970)

[12] A. A. Movchan, The direct method of Liapunov in stability of elastic systems, Prik. Mat. Mech. 23, 483-394 (1959)

[13] R. H. Plaut and E. F. Infante, On the stability of some continuous systems subjected to random excitation, J. Appl. Mech. 37, 623-628 (1970) 